\title{
A comparison of curated gene sets versus transcriptomics-derived gene signatures for detecting pathway activation in immune cells
}

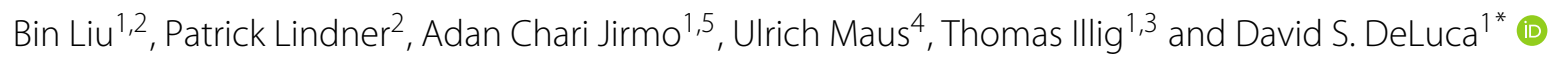

\begin{abstract}
Background: Despite the significant contribution of transcriptomics to the fields of biological and biomedical research, interpreting long lists of significantly differentially expressed genes remains a challenging step in the analysis process. Gene set enrichment analysis is a standard approach for summarizing differentially expressed genes into pathways or other gene groupings. Here, we explore an alternative approach to utilizing gene sets from curated databases. We examine the method of deriving custom gene sets which may be relevant to a given experiment using reference data sets from previous transcriptomics studies. We call these data-derived gene sets, "gene signatures" for the biological process tested in the previous study. We focus on the feasibility of this approach in analyzing immune-related processes, which are complicated in their nature but play an important role in the medical research.

Results: We evaluate several statistical approaches to detecting the activity of a gene signature in a target data set. We compare the performance of the data-derived gene signature approach with comparable GO term gene sets across all of the statistical tests. A total of 61 differential expression comparisons generated from 26 transcriptome experiments were included in the analysis. These experiments covered eight immunological processes in eight types of leukocytes. The data-derived signatures were used to detect the presence of immunological processes in the test data with modest accuracy (AUC $=0.67)$. The performance for $\mathrm{GO}$ and literature based gene sets was worse $(\mathrm{AUC}=$ 0.59). Both approaches were plagued by poor specificity.

Conclusions: When investigators seek to test specific hypotheses, the data-derived signature approach can perform as well, if not better than standard gene-set based approaches for immunological signatures. Furthermore, the data-derived signatures can be generated in the cases that well-defined gene sets are lacking from pathway databases and also offer the opportunity for defining signatures in a cell-type specific manner. However, neither the data-derived signatures nor standard gene-sets can be demonstrated to reliably provide negative predictions for negative cases. We conclude that the data-derived signature approach is a useful and sometimes necessary tool, but analysts should be weary of false positives.
\end{abstract}

Keywords: Transcriptome, Gene signature, Gene set

\footnotetext{
*Correspondence: DeLuca.David@mh-hannover.de

†Equal contributor

${ }^{1}$ Hannover Medical School, Biomedical Research in Endstage and Obstructive

Lung Disease Hannover (BREATH), German Center for Lung Research,

Carl-Neuberg-Straße, 30625 Hannover, Germany

Full list of author information is available at the end of the article
}

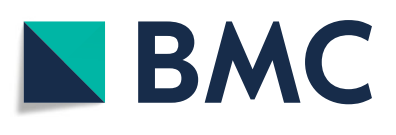

(c) The Author(s). 2020 Open Access This article is distributed under the terms of the Creative Commons Attribution 4.0 International License (http://creativecommons.org/licenses/by/4.0/), which permits unrestricted use, distribution, and reproduction in any medium, provided you give appropriate credit to the original author(s) and the source, provide a link to the Creative Commons license, and indicate if changes were made. The Creative Commons Public Domain Dedication waiver (http://creativecommons.org/publicdomain/zero/1.0/) applies to the data made available in this article, unless otherwise stated. 


\section{Background}

With the advent of high-throughput sequencing technology, transcriptome data sets are being generated on a massive scale. Differential expression analyses produce long lists of genes, requiring summarization approaches to allow for the biological interpretation of the results. Gene Set Enrichment Analysis (GSEA) [1], Over-Representation Analysis (ORA) and Gene Set Analysis (GSA, also referred to as Pathway Analysis) have been developed to this end. These methods rely on catalogues of gene sets which are associated with various biological processes, relying either on literature or high throughput experimentation. By applying statistics such as the MannWhitney-Wilcoxon Test, Fisher's Exact Test [2] or the Kolmogorov-Smirnov statistic [1], these methods enable one to interpret the relevance of a biological process in a given experiment. The curated gene sets are archived in a range of biological pathway databases including the Gene Ontology (GO) [3], the KEGG (Kyoto Encyclopedia of Genes and Genomes) Pathway database [4] and The Reactome Knowledgebase [5].

Although GSEA, ORA and GSA have been widely adopted to interpret the results of transcriptomics studies, their value is limited by the number of curated gene sets available to the researchers. In many cases, researchers may fail to find curated gene sets from such databases best describing the complex immunological process they are most interested in. In other cases, curated gene sets may reflect cell-type specificity, and could involve multiple cell types. This creates a limitation in applicability to expression based studies in which a single cell type is profiled.

Meanwhile, the construction of repositories for archiving transcriptomic data sets enables one to access high-quality data generated by previous studies. Gene Expression Omnibus (GEO) from the National Center for Biotechnology Information (NCBI) [6] and ArrayExpress from European Molecular Biology Laboratory (EMBL) $[7,8]$ are two prominent examples among many. Given this wealth of resources, we examine a data-derived signatures approach. We evaluated the simple methodology of testing whether a given biological process is activated in a target data set by deriving a relevant gene signature from a previous transcriptomics experiment and testing the presence of that signature in the target data set. Deriving gene sets directly from previous transcriptomics experiments has several precedents, for example portions of MSigDB $[9,10]$. Here, we focus our analysis in the context of immune cells. We evaluate several statistical approaches to detecting the activity of a gene signature in a target data set, some of which are previously used in gene set enrichment analysis such as Wilcoxon Test, and Fisher Exact Test, as well as a novel expression concordance score test. We compare the performance of the data-derived gene signature approach with comparable GO term gene sets across all of the statistical tests.

\section{Methods}

All the tests in this section were processed using $\mathrm{R}$ (Version 3.2.3) on platform x86_64-pc-linux-gnu (64-bit) [11] embedded in RStudio (Version 1.1.463) [12].

\section{Data source}

We downloaded 25 immune-related Series (GSE) from GEO repository in the Series Matrix File form, 22 of which are describing significantly activated biological processes and will be used for generation of the signatures or true positive targets for validation. The remaining three were used as negative targets for assessing specificity. T Nikolic et al. supplied the analyzed data of their publication [13], which systematically profiled the differentially expressed genes (DEGs) between tolerogenic dendritic cells (tolDCs) and non-modulated mature inflammatory dendritic cells (mDCs). A brief description of each data set is given in Table 1.

\section{Signature generation}

We consider a signature to be a collection of genes whose expression changes in association with a specific cellular process. The signatures to be detected were generated by the following two strategies:

1) Generation of data-derived signature and target data sets: We downloaded 28 immune-related Series (GSE) from the GEO repository in the Series Matrix File form, 25 of which describe activated biological processes. $\mathrm{T}$ Nikolic et al. supplied the analyzed data of their publication [13], which systematically profiled the differentially expressed genes (DEGs) between tolerogenic dendritic cells (tol-DCs) and non-modulated mature inflammatory dendritic cells (mDCs).

To access specificity, negative target data sets are required. The main approach we take is to simply consider for a given immunological process, the target data sets of the remaining immunological processes in our study to be negative cases. We also provide an alternative approach to defining negative cases in which the control samples from Series GSE101710, GSE110223, and GSE21045 were randomly selected and equally distributed into two different groups, representing pseudo-phenotypes for differential expression.

Both the data-derived signatures and target datasets are based on differential expression (DE) analysis. The Differential expression of microarray data were analyzed in $\mathrm{R}$ using limma (Version 3.26.9) [40]. The RNA-seq data of GSE112899, GSE73213, GSE111789 and GSE128027 were analyzed in $\mathrm{R}$ using DESeq2 package (Version 1.24.0) [41]. $P$-values of the DE (differential expression) analysis were corrected for multiple tests using the q-value method [42]. 
Table 1 Transcriptomics data sources

\begin{tabular}{|c|c|c|c|}
\hline ID & Experiment design & Experimental Organism & Contributor \\
\hline tolerogenic_DC & Tolerogenic DC & Homo Sapiens & T Nikolic et al. [13] \\
\hline GSE17721 & Tolerogenic DC & Mus Musculus & | Amit et al. [14] \\
\hline GSE18921 & Tolerogenic DC & Homo Sapiens & H Torres-Aguilar et al. [15] \\
\hline GSE5099 & Monocytes differentiation & Homo Sapiens & FO Martinez et al. [16] \\
\hline GSE8286 & Monocytes differentiation & Homo Sapiens & H Liu et al. [17] \\
\hline GSE111475 & B cell activation & Homo Sapiens & K Miyawaki et al. \\
\hline GSE116999 & B cell activation & Homo Sapiens & DT Avery et al. [18] \\
\hline GSE51587 & B cell activation & Homo Sapiens & ᄂ Berglund et al. [19] \\
\hline GSE54017 & B cell activation & Homo Sapiens & A Shimabukuro-Vornhagen et al. [20] \\
\hline GSE29797 & $\mathrm{T}$ cell activation & Mus Musculus & Yang K et al. [21] \\
\hline GSE112899 & T cell activation & Homo Sapiens & Sousa IG et al. [22] \\
\hline GSE60235 & T cell activation & Homo Sapiens & Ye CJ et al. [23] \\
\hline GSE73213 & T cell activation & Homo Sapiens & LaMere SA et al. [24, 25] \\
\hline GSE111789 & Eosinophils cytokine response & Homo Sapiens & Khoury P et al. \& Gadkari M et al. [26, 27] \\
\hline GSE112010 & Eosinophils cytokine response & Mus Musculus & Fairfax KA et al. [28] \\
\hline GSE128027 & Eosinophils cytokine response & Homo Sapiens & Nelson RK et al. [29] \\
\hline GSE104152 & Naive to Th17 differentiation & Mus Musculus & Mohammad I et al. [30] \\
\hline GSE113889 & Naive to Th17 differentiation & Homo Sapiens & Tangye S et al. (Accession: GSE113889) \\
\hline GSE1 18974 & Naive to Th17 differentiation & Homo Sapiens & Tripathi SK et al. [31] \\
\hline GSE140443 & Naive to Th17 differentiation & Mus Musculus & Gehrmann U et al. (Accession: GSE140443) \\
\hline GSE110446 & NKIL12 & Homo Sapiens & Costanzo MC et al. [32] \\
\hline GSE24791 & NKIL12 & Homo Sapiens & Campbell AR et al. [33] \\
\hline GSE63038 & NKIL12 & Homo Sapiens & de Carvalho EG et al. (Accession: GSE63038) \\
\hline GSE87290 & PBMC LPS & Homo Sapiens & Lin J et al. [34] \\
\hline GSE22248 & PBMC LPS & Homo Sapiens & Pena OM et al. [35] \\
\hline GSE9916 & PBMC LPS & Homo Sapiens & Wong HR et al. [36] \\
\hline GSE101710 & Negative data & Homo Sapiens & Zapata HJ et al. [37] \\
\hline GSE110223 & Negative data & Homo Sapiens & Vlachavas El et al. [38] \\
\hline GSE21045 & Negative data & Homo Sapiens & Landolin JM et al. [39] \\
\hline
\end{tabular}

For each immunological process in our study, we obtained multiple GEO series, one of which we selected for signature generation and the remaining experiments represent the target data sets. We then iterated the target series, and selected each of them a signature set, such that each experiment was considered to be a target set and a signature set at one point. We also assessed the impact of selecting the "best" experiment as the signature data to determine whether being selective in choosing the signature set could be beneficial. Here the "highest quality" is defined by making a judgment based on the sample size, platform, and specifics about the experimental design.

2) Selection of curated gene sets: For each of the relevant cellular processes captured by the data-derived signature, we searched the literature and gene set databases (GO, KEGG) for appropriate gene sets. We included a literature-based tolerogenic DC (dendritic cell) signature as proposed by $\mathrm{C}$ Orabona et al. [43]. No comparable tolerogenic DC signatures were available in the KEGG or GO databases. We also merged the annotation list of GO term Positive Regulation of Monocyte Differentiation (GO:0045657) and Negative Regulation of Monocyte Differentiation (GO:0045656) as the curated gene set for Monocyte differentiation and Positive Regulation of $\mathrm{B}$ Cell Activation (GO:0050871) and Negative Regulation of B Cell Activation (GO:0050869) as the curated gene set for B cell activation. GO:2000417 Negative Regulation of Eosinophil Migration and GO:2000418 Positive Regulation of Eosinophil Migration was merged for detection of the Eosinophil cytokine response. GO:0032824 and GO:0032825 (Negative/Positive Regulation of Natural Killer Cell Differentiation) together as a merged list 
for NK (natural killer) IL12 (interleukin) stimulation and GO:0034142 (Toll-Like Receptor 4 Signaling Pathway) for PBMC (peripheral blood mononuclear cell) to LPS (lipppolysaccride) response. The curated data set for the detection of Naive to Th17 differentiation derived from a merged list of GO:0050868 and GO:0050870 (Negative/Positive Regulation of $\mathrm{T}$ helper-17 cell differentiation) and $\mathrm{T}$ cell activation described by merging the GO annotation of GO:0050868 and GO:0050870 (Negative/Positive Regulation of $\mathrm{T}$ cell activation). The list of curated gene sets utilized as the signature in this approach is found in Table 2.

\section{Signature detection}

We would like to know whether a cellular process represented by a signature has been deferentially regulated in a target experiment. To detect the presence of the signatures in the target data sets, we applied the following alternative methods:

1) The Mann-Whitney-Wilcoxon Enrichment Test: We used Mann-Whitney-Wilcoxon Test in the same manner found in the PANTHER [2] webtool. Genes are ranked by their fold change in the target data set. The test is then performed between the ranks of the signature genes versus the ranks of the non-signature genes. $P$-values are provided by the wilcox.test() function from $\mathrm{R}$ stats package [44].

2) Fisher's Exact Overrepresentation Test: The Fisher's Exact Test is applied in a comparable fashion to tool such as PANTHER [2]. The test is based on a contingency table comparing the differential expression status (DE or not $\mathrm{DE}$ ) vs the signature status (in signature or not in signature). A demonstration of the contingency table is available in Table 3 below. $P$-values were generated by the fisher.test() function from R stats [44].

3) Correlation Permutation: This method is a quantitative permutation test based on Spearman's rank correlation. This correlation is calculated for the signature genes between their ranks in the signature and their ranks in the target data set based on fold change. In performing permutations, we randomly selected a gene set with the same length as the signature the set of genes common to the two data sets. The Spearman correlation was calculated for each of 10,000 permutation, giving an empirical distribution from which p-value was derived. The sign of the correlation represented the direction of the signature in the target set.

4) Concordance Permutation: The signature concordance Test is a semi-quantitative permutation test based on the regulation directions. For the test, a concordance score is defined as:

concordance score $=\frac{N_{\text {signature genes matching in regulation directions }}}{N_{\text {genes in the signature }}}$

where $N$ refers to the number of genes.

The concordance score of the signature was calculated using Equation 1. A total of 10,000 permutations were performed to generate an empirical null distribution. In each permutation, we randomly selected a gene set with the same length as the signature from all the genes which are in common between the signature-generating platform and the target data set platform and record the concordance score. $P$-values are derived from the test statistic and the empirical null distribution. The signature direction is determined by whether the concordance score of

Table 2 Currated gene sets

\begin{tabular}{lll}
\hline Name & Description & Process \\
\hline GO:0050868 & Negative regulation of T cell activation 0050870 & Tcell_activation \\
GO:2000320 & Positive regulation of T cell activation & Tcell_activation \\
GO:2000321 & Negative & Regulation of T helper-17 cell differentiation \\
GO:0032824 & Positive & Regulation of T helper-17 cell differentiation \\
GO:0032825 & Negative regulation of natural killer cell differentiation & NK_IL12 \\
GO:2000417 & Positive regulation of natural killer cell differentiation & NK_IL12 \\
GO:2000418 & Negative regulation of eosinophil migration & Eosinophils_cytokine_response \\
GO:0045657 & Positive regulation of eosinophil migration & Eosinophils_cytokine_response \\
GO:0045656 & Positive regulation of Monocyte Differentiation & Monocyte_Macrophage_differentiation \\
GO:0050871 & Negative regulation of Monocyte Differentiation & Monocyte_Macrophage_differentiation \\
G0:0050869 & Positive regulation of B cell activation & Bcell_activation \\
GO:0034142 & Negative regulation of B cell activation & Bcell_activation \\
tolerogenic DC signature & Toll-like receptor 4 signaling pathway & PBMC_LPS \\
\hline
\end{tabular}


Table 3 A comparison of the contingency table for Concordance Fisher's Exact (above) and Fisher's Exact Overrepresentation (below)

\begin{tabular}{llll}
\hline & Genes matched in regulation direction & Genes not matched in regulation direction & Total \\
In signature & $x$ & $m-x$ & $m$ \\
Not in signature & $k-x$ & $n-(k-x)$ & $n$ \\
Total & $k$ & $m+n-k$ & $m+n$ \\
& Differentially expressed genes & Not differentially expressed genes & Total \\
In signature & $x$ & $m-x$ & $m$ \\
Not in signature & $k-x$ & $n-(k-x)$ & $m+n$ \\
Total & $k$ & $m+n-k$ & $m$ \\
\hline
\end{tabular}

the signature is greater or less than the mean of permuted scores.

5) Concordance Fisher's Exact Test: As in the previous method, this method is also based on the concordance of the fold change direction, however with a different strategy for deriving a p-value. Here, we derive a p-value using the Fisher Exact Test based on the contingency table in Table 3.

It should be noted that the first two tests (overrepresentation and enrichment) consider the gene signature to be an unordered set of genes, and the resulting test does not provide a direction as to the regulation of the biological process. This is equally applicable to gene signatures and currated gene sets. However, the remaining correlation and concordance tests also provide a direction, and we considered correct direction to be a requirement for true positive / true negative results when accessing the accuracy. Furthermore, these tests require a ranking of the genes in the gene signature, which are not available for currated gene sets, and therefore these tests were not applied to the curated gene sets.

$P$-values of all the five methods were calculated according to the aforementioned description. ROC (receiver operating characteristic) curves were generated by changing the confidence level threshold (the threshold for $p$ values) for the logistic classification of whether the signature is presented in each target set. The AUC (area under the curve) of each condition was calculated for the comparison.

\section{Results}

We applied all the five methods on data-derived signatures to detect the presence of the signatures in the target data sets and to evaluate the performance of the methods on data-derived signatures. The ROC curves are summarized in Fig. 1, with AUC under each condition labelled separately. The full table of $p$-values for each target signature combination is provided in the supplement [Additional file 1].

The data derived signature tests performed more favorably than the curated gene set definitions, with the best performance being that of the Fisher Exact Overrepresentation Test for the data derived signature (AUC = 0.67). The Mann-Whitney-Wilcoxon Enrichment test performed poorly (AUC $=0.55)$ for both data-derived and curated gene sets.

While the ROC curve is useful for summarizing performance using a sliding threshold, given that these scores are based on $p$-values, it makes sense to example the specific ubiquitous threshold of 0.05 . The sensitivity and specificity values for an alpha threshold of 0.05 are summarized in Table 4:

\section{Robustness analysis}

We assessed the behavior of these statistics in the presence of increasing noise in the signatures. This was done by replacing a batch of the most significant with the least significant genes, and iteratively increasing the batch size. The effect of noise on the True Positive Rate is showing in Fig. 2. The full table of results is provided in the supplement [Additional file 2].

The robustness analysis shows that the analytical statistics do show a sharp decline in True Positive Rate with the introduction of only 10 percent noise. In contrast, the permutation-based methods show a more gradual effect with increasing noise.

Among the various experiments in our study, we can expect various levels of "noise" also in the sense that the quality of the experiments could be quite heterogeneous. We have accessed the effect of being selective in choosing our signature-generating data sets and calculated the performance when using only the highest quality experiments for generating the signatures (based on sample size, experimental design, platform). The result is an increase in AUC (Fig. 3). This selectivity boosts the AUCs above 0.72 for several statistics, indicating the value of basing signatures on high quality experiments.

\section{Specificity analysis}

Faced with the need to define a set of target data sets as negative cases, we have opted to consider for a given signature of an immunological process the negative cases to 


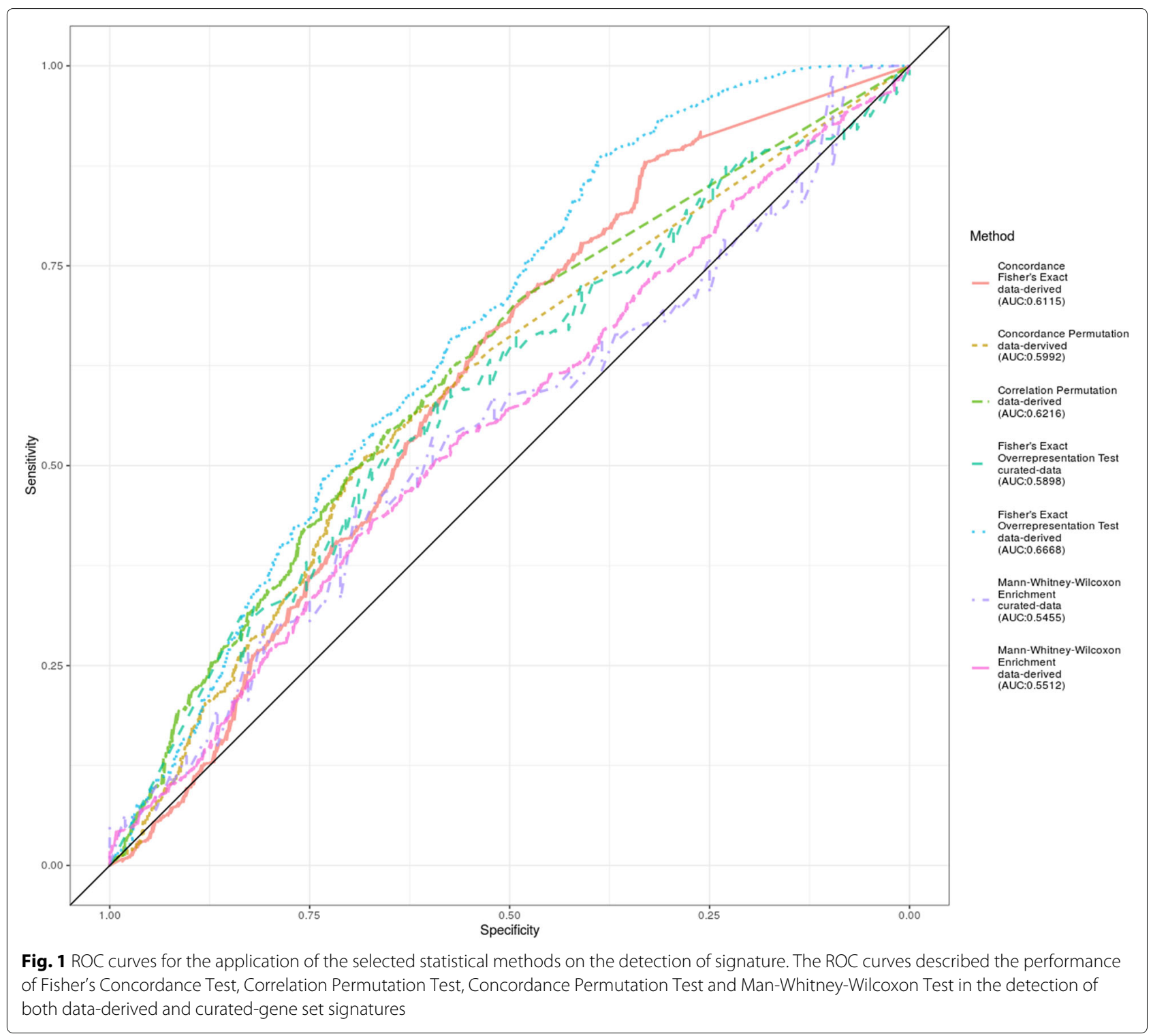

be the target data sets for the other immunological processes which we have collected. However, motivated by the fact that there will be overlapping genes involved in the immunological processes we include here (e.g. genes that are upregulated both during B cell and T cell stimulation), we also provide an alternative definition of negative cases. We have taken control samples from three additional studies and generated a series of random combinations of samples into pseudo-phenotypes for differential expression analysis. Using this definition of negative samples, we

Table 4 The sensitivity and the specificity of the five methods at an alpha cutoff of 0.05

\begin{tabular}{|c|c|c|c|c|}
\hline \multirow{2}{*}{$\begin{array}{l}\text { Signature Generation } \\
\text { Method }\end{array}$} & \multicolumn{2}{|c|}{ Data-derived signature } & \multicolumn{2}{|c|}{ Curated gene set } \\
\hline & Sensitivity & Specificity & Sensitivity & Specificity \\
\hline Mann-Whitney-Wilcoxon Enrichment & 0.7655 & 0.3098 & 0.25 & 0.7292 \\
\hline Fisher's Exact Concordance & 0.7977 & 0.3720 & - & - \\
\hline Fisher's Exact Overrepresentation & 0.8981 & 0.1014 & 0.5246 & 0.6229 \\
\hline Correlation Permutation & 0.7278 & 0.4517 & - & - \\
\hline Concordance Permutation & 0.7424 & 0.3995 & - & - \\
\hline
\end{tabular}




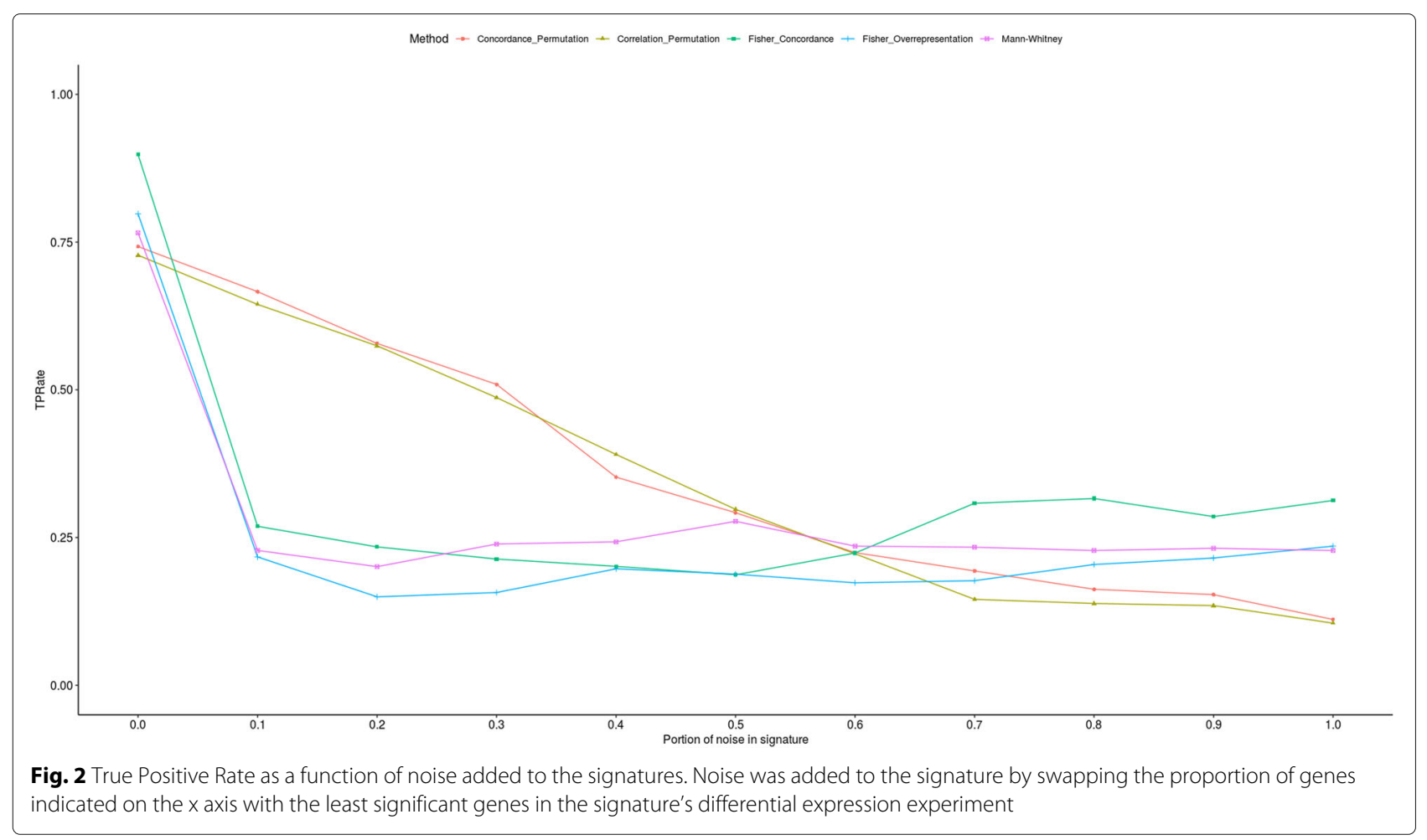

observe the ROC curves in Fig. 4. This type of assessment does lead to higher AUC scores due to the fact that the top DE genes in the pseudo phenotpyes are completely independent of the processes at play in the signature experiments.

To examine the sources of non-specific signature genes, we identified genes that deferentially expressed across most of the signatures. We selected genes whose median absolute fold change was greater than 1 across all the signatures. These genes are provided in supplement [Additional file 3] . Performing a GO enrichment analysis with PANTHER, we see that these genes are common to a wide range of immunological processes [Additional file 3].

\section{Discussion}

We have evaluated methods for detecting the activation or deactivation of immunological processes within target differential expression experiments. We compared a strategy based on gene signatures derived from previous transcriptome experiments with the approach more commonly taken using curated gene sets. The results show that the use of such "custom" gene signatures is a valid approach, despite the fact that they are derived from single experiments, whereas curated gene sets can be based on many sources of information. Overall both methods tend to produce high rates of false positives.

This observation of an abundance of false positives is consistent with previous studies. Tarca et al have taken a similar approach to evaluating their gene set enrichment tool along with other published methods by defining a test data set that consisted of previous DE experiments [45]. They generated signatures for various cancer types and tested them in matching and mismatching cancer target sets. The false positive results were so prevalent that they took the strategy of using the rank of the correct target set in the list of all tested sets to define their performance metric. In other words, the best method was the one that had the fewest false positives of higher rank than the correct target.

Although we evaluate the performance of these methods using AUC, it is important to note that in practice a p-value cutoff of 0.05 is typically used when deciding whether a gene set is differentially expressed. At this cutoff the data-derived signature method exhibits high sensitivity and low specificity. In practice, this method would benefit from a more stringent p-value threshold.

When comparing the various statistical tests among themselves, the Mann-Whitney-Wilcoxon notably underperforms. For the data-derived signatures, at the p-value threshold of 0.05 , the performance is characterized by poor specificity for data-derived signatures and poor sensitivity for curated gene sets. Given the large size of the gene signatures, the test is perhaps very sensitive to even small amounts of bias, resulting in many false positive calls. For the smaller curated gene sets, the method seems to be somewhat more appropriate.

When applying the data-derived gene signatures, we tested two groups of statistics: ones which utilize the 


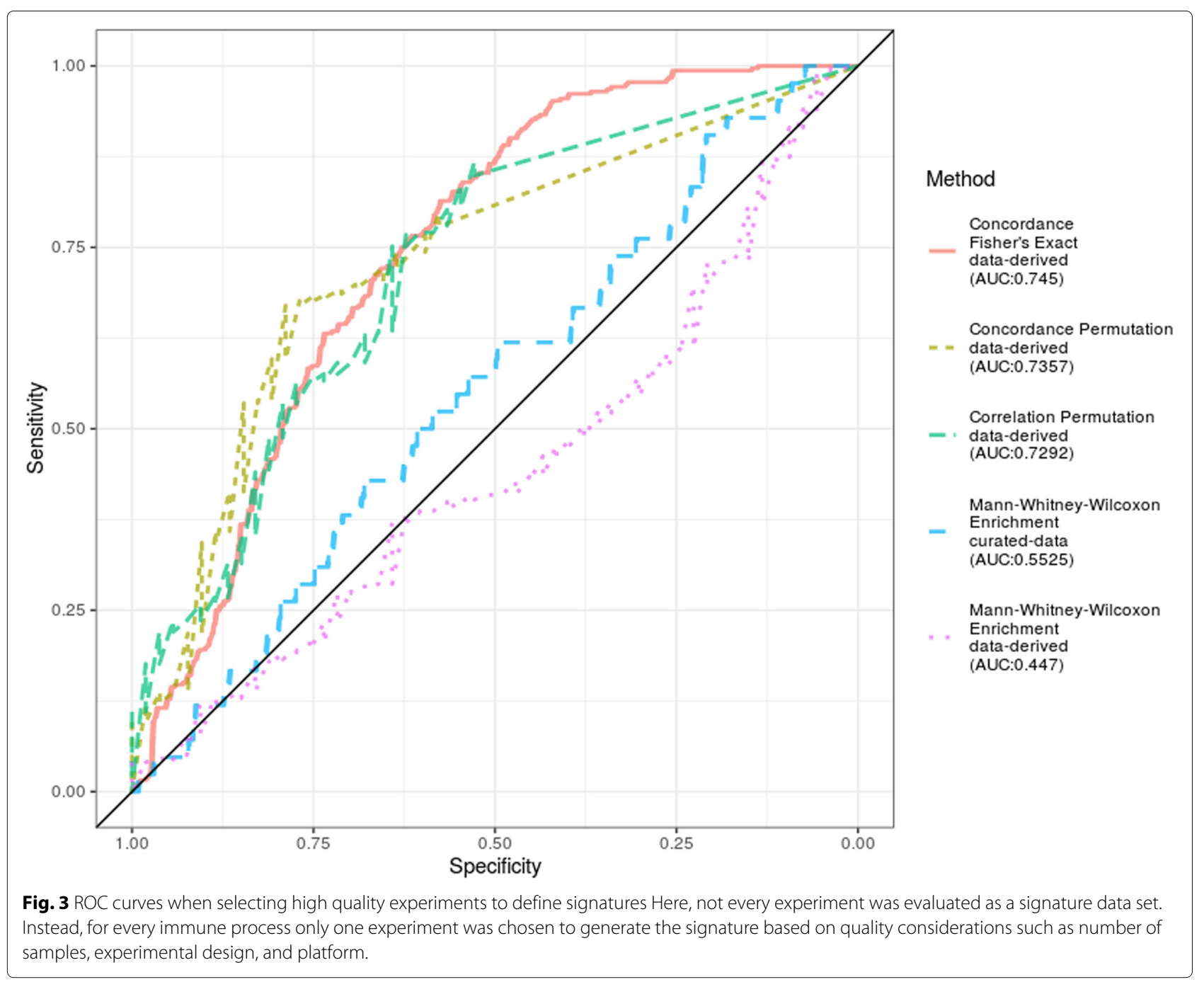

direction of the fold change in the signature data set, and those which use the signatures simply as a set without regard to the direction of change in expression. These results are somewhat ambiguous as to whether there is an advantage to using this additional information, given that one of the two techniques which do not use it performed quite well - namely the Fisher Exact Overrepresentation.

In principle, the a compendium of data derived gene signatures could be generated exhaustively for all of GEO. The MSigDB has taken steps in this direction with for example the $\mathrm{C} 7$ collection of immunological signatures. These collections do contain genes and their change in direction, however information is missing concerning how many genes are in common between the signaturegenerating platform and the target data set platform a discrepancy which will only increase over time with the introduction of new technologies. An additional issue is that these signatures are limited to 200 genes per direction, which is smaller than our data-derived signatures and in our hands shortening the signatures to this length decreases accuracy. There is also a cost trade off between running an "everything against everything" analysis, as is the case in $\mathrm{C} 7$ versus careful manual differential expression analysis by an expert, involving quality control steps and consideration of covariates, batch effect, etc. The former does allow for hypothesis free analysis, however at the expense of many inapplicable tests that reduce power when it comes to multiple test correction.

\section{Conclusion}

In conclusion, the data-derived gene signature approach is a valid and useful tool for inferring the presence and absence of immunological processes in transcriptome datasets. The approach makes valuable use of previously published experiments, and can be carefully tailored to ensure that the most relevant comparisons are made when using a hypothesis driven technique. The accuracy is reasonable when compared to the gene set based approach, but both approaches are prone to false positives. The 


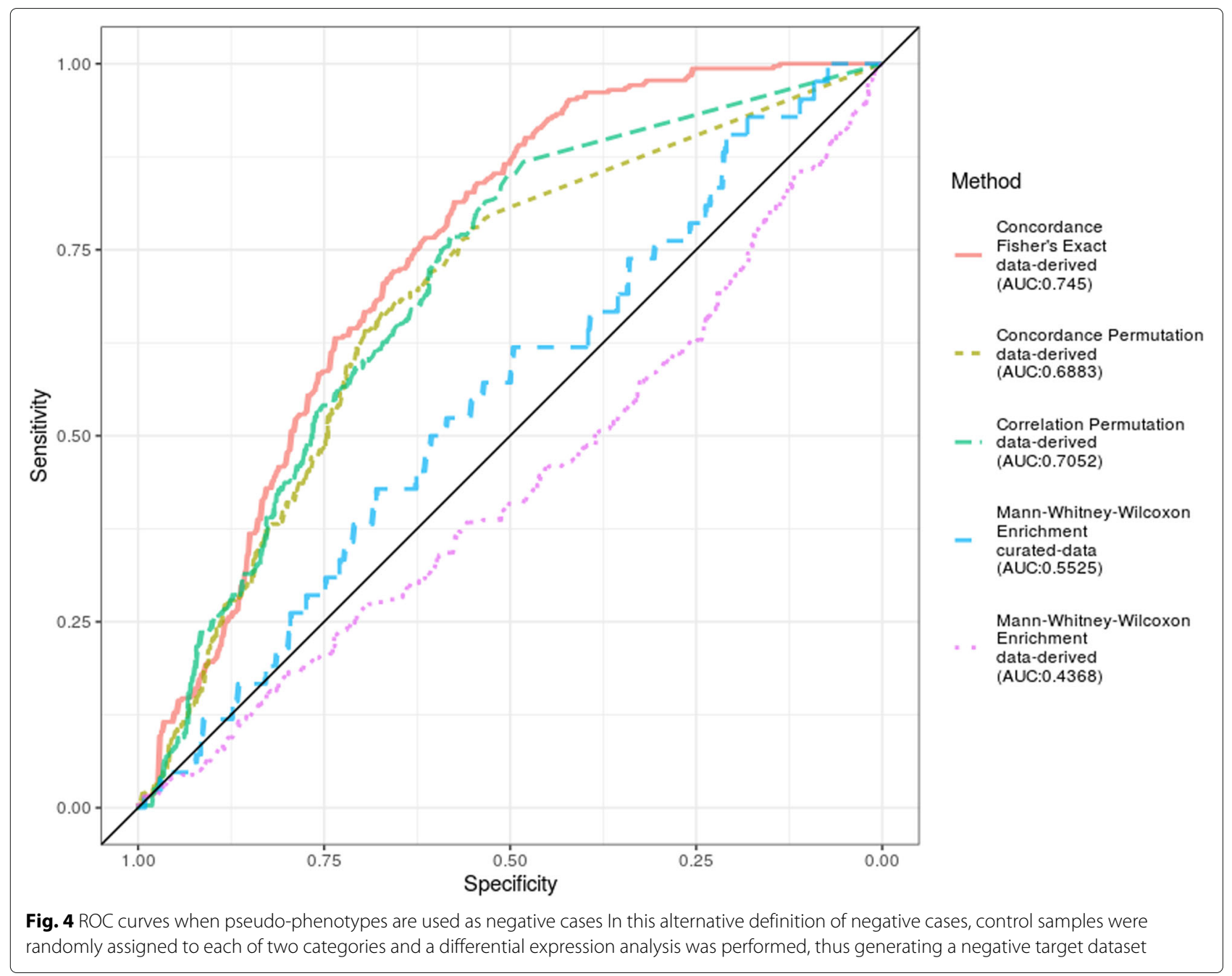

weakness in widely used gene set based approaches is overlooked, perhaps due to the difficulty in producing ground truth information, but this is an issue that must be addressed to improve the interpretation of transcriptome experiments.

\section{Supplementary information}

Supplementary information accompanies this paper at https://doi.org/10.1186/s12859-020-3366-4.

Additional file 1: All Evaluation Results Description: Contains the results for all combinations of signatures, target data sets, and alternative statistical methods. Each sheet represents a statistical method. Each row contains a signature, a target and a series of values that are specific to each of the different approaches.

Additional file 2: Robustness Analysis Description: Results of the robustness analysis when adding noise to the signature. "Portion" refers to what percentage of genes were swapped and the remaining columns are the metrics and outcomes for the various statistical approaches.

Additional file 3: Common Signature Genes and Enrichment Results Description: These are the PANTHER GO analysis results for the set of genes found to be in common among all data-derived signatures.

\section{Abbreviations}

AUC: Area under the curve; DC: Dendritic cell; DE: Differential expression; DEG: Differentially expressed gene; EMBL: European molecular biology laboratory; GEO: Gene expression omnibus; GO: Gene ontology; GSA: Gene set analysis; GSEA: Gene set enrichment analysis; IL: Interleukin; KEGG: Kyoto encyclopedia of genes and genomes; LPS: Lipppolysaccride; mDC: Mature inflammatory dendritic cell; NCBI: National center for biotechnology information; NK: Natural killer; ORA: Over-representation analysis; PBMC: Peripheral blood mononuclear cell; ROC: Receiver operating characteristic; tol-DC: Tolerogenic dendritic cell

\section{Acknowledgements}

We thank Dr. Tatjana Nikolic from Leiden University Medical Centre for sharing us the valuable RNA-seq results for tolerogenicity analysis. Thank you to Dr. Victor A. Farutin for reading the manuscript and sharing his valuable opinions with us.

\section{Authors' contributions}

DSD and BL conceived of the presented idea and developed the theory. BL performed the computations. AJ and UM contributed part of the data and independently verified the analytical methods with their observation in experiments. DSD, TI and PL encouraged BL to investigate the application of data-derived signature and supervised the findings of this work. BL and DSD drafted the paper. All authors discussed the results and contributed to the final manuscript. All authors read and approved the final manuscript. 


\section{Funding}

This work was funded by the Bundesministerium für Bildung und Forschung BMBF (German Center for Lung Research (DZL)). The funding bodies were not involved in the design of the study, collection, analysis, and interpretation of data, nor in writing the manuscript.

\section{Availability of data and materials}

The data sets involved in this study include the following previously published experiments hosted by GEO: GSE17721, GSE18921, GSE5099, GSE8286, GSE111475, GSE116999, GSE51587, GSE54017, GSE29797, GSE112899, GSE60235, GSE73213, GSE111789, GSE140443, GSE110446, GSE24791, GSE63038, GSE87290. GSE22248, GSE9916, GSE101710, GSE110223, GSE21045. The DC tolerogenicity signature data set was provided by Dr. Tatjana Nikolic upon request.

\section{Ethics approval and consent to participate}

Not applicable.

\section{Consent for publication}

Not applicable.

\section{Competing interests}

The authors declare that they have no competing interests.

\section{Author details}

${ }^{1}$ Hannover Medical School, Biomedical Research in Endstage and Obstructive Lung Disease Hannover (BREATH), German Center for Lung Research, CarlNeuberg-Straße, 30625 Hannover, Germany. ${ }^{2}$ Institute of Technical Chemistry, Leibniz University of Hannover, Callinstraße 5, 30167 Hannover, Germany. ${ }^{3}$ Hannover Unified Biobank, Hannover Medical School, Feodor-Lynen-Straße, 30625 Hannover, Germany. ${ }^{4}$ Division of Experimental Pneumology, Hannover Medical School, Feodor-Lynen-Straße 21, 30625 Hannover, Germany. ${ }^{5}$ Department of Pediatric Pneumology,Allergology and Neonatology, Hannover Medical School, Carl-Neuberg-Straße 1, 30625 Hannover, Germany.

Received: 7 October 2019 Accepted: 14 January 2020

Published online: 28 January 2020

\section{References}

1. Subramanian A, Tamayo P, Mootha VK, Mukherjee S, Ebert BL, Gillette MA, Paulovich A, Pomeroy SL, Golub TR, Lander ES, et al. Gene set enrichment analysis: a knowledge-based approach for interpreting genome-wide expression profiles. Proc Natl Acad Sci. 2005;102(43): 15545-50.

2. Mi H, Muruganujan A, Ebert D, Huang X, Thomas PD. Panther version 14: more genomes, a new panther go-slim and improvements in enrichment analysis tools. Nucleic Acids Res. 2018;47(D1):419-26.

3. Ashburner M, Ball CA, Blake JA, Botstein D, Butler H, Cherry JM, Davis AP, Dolinski K, Dwight SS, Eppig JT, et al. Gene ontology: tool for the unification of biology. Nature Genet. 2000;25(1):25.

4. Kanehisa M, Goto S. Kegg: kyoto encyclopedia of genes and genomes. Nucleic Acids Res. 2000;28(1):27-30.

5. Croft D, Mundo AF, Haw R, Milacic M, Weiser J, Wu G, Caudy M Garapati P, Gillespie M, Kamdar MR, et al. The reactome pathway knowledgebase. Nucleic Acids Res. 2013;42(D1):472-7.

6. Barrett T, Wilhite SE, Ledoux P, Evangelista C, Kim IF, Tomashevsky M, Marshall KA, Phillippy KH, Sherman PM, Holko M, et al. Ncbi geo: archive for functional genomics data sets-update. Nucleic Acids Res. 2012;41(D1):991-5.

7. Brazma A, Parkinson $H$, Sarkans U, Shojatalab M, Vilo J, Abeygunawardena N, Holloway E, Kapushesky M, Kemmeren P, Lara $\mathrm{GG}$, et al. Arrayexpress - a public repository for microarray gene expression data at the ebi. Nucleic Acids Res. 2003;31(1):68-71.

8. Athar A, Füllgrabe A, George N, lqbal H, Huerta L, Ali A, Snow C, Fonseca NA, Petryszak R, Papatheodorou l, et al. Arrayexpress update-from bulk to single-cell expression data. Nucleic Acids Res. 2018;47(D1):711-5.

9. Liberzon A, Subramanian A, Pinchback R, Thorvaldsdóttir H, Tamayo P, Mesirov JP. Molecular signatures database (msigdb) 3.0. Bioinformatics. 2011;27(12):1739-40.

10. Godec J, Tan Y, Liberzon A, Tamayo P, Bhattacharya S, Butte AJ, Mesirov JP, Haining WN. Compendium of immune signatures identifies conserved and species-specific biology in response to inflammation. Immunity. 2016;44(1):194-206

11. R Core Team. R: A Language and Environment for Statistical Computing Vienna: R Foundation for Statistical Computing; 2018. https://www.Rproject.org/.

12. RStudio Team. RStudio: Integrated Development Environment for R. Boston: RStudio, Inc.; 2016. http://www.rstudio.com/.

13. Nikolic T, Woittiez N, van der Slik A, Laban S, Joosten A, Gysemans C, Mathieu C, Zwaginga J, Koeleman B, Roep B. Differential transcriptome of tolerogenic versus inflammatory dendritic cells points to modulated t1 d genetic risk and enriched immune regulation. Genes Immun. 2017;18(3):176.

14. Amit I, Garber M, Chevrier N, Leite AP, Donner Y, Eisenhaure T, Guttman M, Grenier JK, Li W, Zuk O, et al. Unbiased reconstruction of a mammalian transcriptional network mediating pathogen responses. Science. 2009:326(5950):257-63.

15. Torres-Aguilar H, Aguilar-Ruiz SR, González-Pérez G, Munguía R, Bajaña S, Meraz-Ríos MA, Sánchez-Torres C. Tolerogenic dendritic cells generated with different immunosuppressive cytokines induce antigen-specific anergy and regulatory properties in memory cd4+t cells. J Immunol. 2010;184(4):1765-75.

16. Martinez FO, Gordon S, Locati M, Mantovani A. Transcriptional profiling of the human monocyte-to-macrophage differentiation and polarization: new molecules and patterns of gene expression. J Immunol. 2006;177(10):7303-11.

17. Liu H, Shi B, Huang C-C, Eksarko P, Pope RM. Transcriptional diversity during monocyte to macrophage differentiation. Immunol Lett. 2008;117(1):70-80.

18. Avery DT, Kane A, Nguyen T, Lau A, Nguyen A, Lenthall H, Payne K, Shi W, Brigden $\mathrm{H}$, French $\mathrm{E}$, et al. Germline-activating mutations in pik3cd compromise b cell development and function. J Exp Med. 2018;215(8): 2073-95.

19. Berglund LJ, Avery DT, Ma CS, Moens L, Deenick EK, Bustamante J, Boisson-Dupuis S, Wong M, Adelstein S, Arkwright PD, et al. II-21 signalling via stat3 primes human naive $b$ cells to respond to il-2 to enhance their differentiation into plasmablasts. Blood. 2013;122(24): 3940-50.

20. Shimabukuro-Vornhagen A, Zoghi S, Liebig TM, Wennhold K, Chemitz J, Draube A, Kochanek M, Blaschke F, Pallasch C, Holtick U, et al. Inhibition of protein geranylgeranylation specifically interferes with cd40-dependent b cell activation, resulting in a reduced capacity to induce t cell immunity. J Immunol. 2014;193(10):5294-305.

21. Yang K, Neale G, Green DR, He W, Chi H. The tumor suppressor tsc1 enforces quiescence of naive t cells to promote immune homeostasis and function. Nat Immunol. 2011;12(9):888.

22. Sousa IG, Simi KCR, do Almo MM, Bezerra MAG, Doose G, Raiol T, Stadler PF, Hoffmann S, Maranhão AQ, Brigido MM. Gene expression profile of human t cells following a single stimulation of peripheral blood mononuclear cells with anti-cd3 antibodies. BMC Genomics. 2019;20(1): 593.

23. Ye CJ, Feng T, Kwon H-K, Raj T, Wilson MT, Asinovski N, McCabe C, Lee $\mathrm{MH}$, Frohlich I, Paik H-i, et al. Intersection of population variation and autoimmunity genetics in human t cell activation. Science. 2014;345(6202):1254665.

24. LaMere SA, Thompson RC, Komori HK, Mark A, Salomon DR. Promoter h3k4 methylation dynamically reinforces activation-induced pathways in human cd4 t cells. Genes Immun. 2016;17(5):283.

25. LaMere SA, Thompson RC, Meng X, Komori HK, Mark A, Salomon DR. H3k27 methylation dynamics during $\mathrm{cd} 4 \mathrm{t}$ cell activation: regulation of jak/stat and il12rb2 expression by jmjd3. J Immunol. 2017;199(9):3158-75.

26. Khoury P, Stokes K, Gadkari M, Makiya M, Legrand F, Hu Z, Klion A, Franco L. Glucocorticoid-induced eosinopenia in humans can be linked to early transcriptional events. Allergy. 2018;73(10):2076-9.

27. Gadkari M, Makiya MA, Legrand F, Stokes K, Brown T, Howe K, Khoury P, $\mathrm{Hu}$ Z, Klion A, Franco LM. Transcript-and protein-level analyses of the response of human eosinophils to glucocorticoids. Sci Data. 2018;5: https://doi.org/10.1038/sdata.2018.275.

28. Fairfax KA, Bolden JE, Robinson AJ, Lucas EC, Baldwin TM, Ramsay KA, Cole R, Hilton DJ, de Graaf CA. Transcriptional profiling of eosinophil subsets in interleukin-5 transgenic mice. J Leukoc Biol. 2018;104(1): 195-204. 
29. Nelson RK, Brickner $H$, Panwar $B$, Ramírez-Suástegui $C$, Herrera-de la Mata S, Liu N, Diaz D, Alexander LEC, Ay F, Vijayanand P, et al. Human eosinophils express a distinct gene expression program in response to il-3 compared with common $\beta$-chain cytokines il-5 and gm-csf. J Immunol. 20191801668. https://doi.org/10.4049/jimmunol.1801668.

30. Mohammad I, Nousiainen K, Bhosale SD, Starskaia I, Moulder R, Rokka A, Cheng F, Mohanasundaram P, Eriksson JE, Goodlett DR, et al. Quantitative proteomic characterization and comparison of t helper 17 and induced regulatory t cells. PLoS Biol. 2018;16(5):2004194.

31. Tripathi SK, Välikangas T, Shetty A, Khan MM, Moulder R, Bhosale SD, Komsi E, Salo V, De Albuquerque RS, Rasool O, et al. Quantitative proteomics reveals the dynamic protein landscape during initiation of human th17 cell polarization. iscience. 2019;11:334-55.

32. Costanzo MC, Kim D, Creegan M, Lal KG, Ake JA, Currier JR, Streeck $H$ Robb ML, Michael NL, Bolton DL, et al. Transcriptomic signatures of nk cells suggest impaired responsiveness in hiv-1 infection and increased activity post-vaccination. Nat Commun. 2018;9(1):1212.

33. Campbell AR, Regan K, Bhave N, Pattanayak A, Parihar R, Stiff AR, Trikha P, Scoville SD, Liyanarachchi S, Kondadasula SV, et al. Gene expression profiling of the human natural killer cell response to fc receptor activation: unique enhancement in the presence of interleukin-12. BMC Med Genomics. 2015;8(1):66.

34. Lin J, Hu Y, Nunez S, Foulkes AS, Cieply B, Xue C, Gerelus M, Li W, Zhang H, Rader DJ, et al. Transcriptome-wide analysis reveals modulation of human macrophage inflammatory phenotype through alternative splicing. Arterioscler Thromb Vasc Biol. 2016;36(7):1434-47.

35. Pena OM, Pistolic J, Raj D, Fjell CD, Hancock RE. Endotoxin tolerance represents a distinctive state of alternative polarization (m2) in human mononuclear cells. J Immunol. 2011;186(12):7243-54.

36. Wong HR, Odoms K, Sakthivel B. Divergence of canonical danger signals: the genome-level expression patterns of human mononuclear cells subjected to heat shock or lipopolysaccharide. BMC Immunol. 2008;9(1): 24.

37. Zapata HJ, Van Ness PH, Avey S, Siconolfi B, Allore HG, Tsang S, Wilson J, Barakat L, Mohanty S, Shaw AC. Impact of aging and hiv infection on the function of the c-type lectin receptor mincle in monocytes. J Gerontol Ser A. 2018;74(6):794-801.

38. Vlachavas E-I, Pilalis E, Papadodima O, Koczan D, Willis S, Klippel S, Cheng C, Pan L, Sachpekidis C, Pintzas A, et al. Radiogenomic analysis of f-18-fluorodeoxyglucose positron emission tomography and gene expression data elucidates the epidemiological complexity of colorectal cancer landscape. Comput Struct Biotechnol J. 2019;17:177-85.

39. Landolin JM, Johnson DS, Trinklein ND, Aldred SF, Medina C, Shulha H, Weng Z, Myers RM. Sequence features that drive human promoter function and tissue specificity. Genome Res. 2010;20(7):890-8.

40. Ritchie ME, Phipson B, Wu D, Hu Y, Law CW, Shi W, Smyth GK. limma powers differential expression analyses for RNA-sequencing and microarray studies. Nucleic Acids Res. 2015;43(7):47.

41. Love MI, Huber W, Anders S. Moderated estimation of fold change and dispersion for rna-seq data with deseq2. Genome Biol. 2014;15:550. https://doi.org/10.1186/s13059-014-0550-8.

42. Storey JD, Tibshirani R. Statistical significance for genomewide studies. Proc Natl Acad Sci. 2003;100(16):9440-5.

43. Orabona C, Puccetti P, Vacca C, Bicciato S, Luchini A, Fallarino F, Bianchi R, Velardi E, Perruccio K, Velardi A, et al. Toward the identification of a tolerogenic signature in ido-competent dendritic cells. Blood. 2006;107(7):2846-54.

44. R Core Team. R: A Language and Environment for Statistical Computing Vienna: R Foundation for Statistical Computing; 2018. https://www.Rproject.org/.

45. Tarca AL, Bhatti G, Romero R. A comparison of gene set analysis methods in terms of sensitivity, prioritization and specificity. PLOS ONE. 2013;8(11): 1-10. https://doi.org/10.1371/journal.pone.0079217.

\section{Publisher's Note}

Springer Nature remains neutral with regard to jurisdictional claims in published maps and institutional affiliations.

Ready to submit your research? Choose BMC and benefit from:

- fast, convenient online submission

- thorough peer review by experienced researchers in your field

- rapid publication on acceptance

- support for research data, including large and complex data types

- gold Open Access which fosters wider collaboration and increased citations

- maximum visibility for your research: over $100 \mathrm{M}$ website views per year

At BMC, research is always in progress.

Learn more biomedcentral.com/submissions 